\title{
COPE Requires Greater Consistency and Accountability
}

\author{
Jaime A. Teixeira da Silva \\ P. O. Box 7, Miki-cho Post Office, \\ Ikenobe 3011-2, Kagawa-ken, 761-0799, Japan
}

Doi:10.5901/mjss.2017.v8n1p11

\begin{abstract}
The Committee on Publication Ethics (COPE) is the largest ethics-related body in the world. However, the lack of clear policy related to the ethics of self-plagiarism, its stated inability to intervene in ethics-related conflicts involving several of its members causing a relative lack of opacity when authors wish to communicate concerns with or about a COPE member, inconsistent use of ethics guidelines by all its members, and the inability to call out its members when these may appear to be violating COPE editorial guidelines all contribute to the decrease in trust that authors - who are clearly not represented by the COPE charter have in this organization and its members. One of the key corrupting factors is money. COPE members pay annual fees to become members, but only editors and publishers can become members. Consequently authors' rights and concerns about COPE members are rarely addressed. Authors, who already have minimal rights in the entire publishing process, and very limited recourse for self-defense or protest, are considerably marginalized should conflicts with a COPE member exist. How then, can authors and the public hold COPE members more accountable?
\end{abstract}

Keywords: conflicts of interest; opacity; openness; power; transparency; trust

The Committee on Publication Ethics (COPE) is a registered charity in the UK (COPE 2016a) whose modus operandi states: "COPE is a forum for editors and publishers of peer reviewed journals to discuss all aspects of publication ethics. It also advises editors on how to handle cases of research and publication misconduct." (COPE 2016b). By November, 2016, COPE boasted 11,337 members, rising from 11,218 members in September, 2016, almost exclusively journals and publishers (COPE 2016c), including the most profitable (Larivière et al. 2015). Membership is not free, and membership fees range from hundreds to tens of thousands of pounds yearly, depending on the number of journals that plan to be members. Since money is involved in the membership equation, the issue of a financial conflict of interest can never be excluded. Sadly, regular scientists and authors cannot become members. This indicates that COPE is an exclusive club that only represents the rights and the interests of its members. Even though COPE is a registered charity, there is no detailed breakdown of the income received from membership, if any members are receiving special discounts, or the costs of operation. The underlying benefits that members derive remain a secret to the public even though the services that are provided in the form of PDF files, case studies and flow-diagrams should be open access and free to the entire scientific community, with financial support by the powerful members to support logistic costs such as site hosting and maintenance. Examination of the newly appointed COPE Council (COPE 2016d) indicates powerful members in powerful organizations such as publishers (see Kahn case below) and editing companies such as the Edanz Group, represented by Dr. Trevor Lane. This indicates that COPE has evolved from an ethics-suggesting body for editors and publishers, to a powerful corporation that provides an ethical mask to equally powerful editing and publishing-related corporations. This powerful structure, with no rival at the global scale, constitutes a monopoly on ethics.

Claims by authors or scientists against COPE members or concerns with COPE members cannot be resolved directly through COPE, and COPE claims to simply serve as a bridge between authors and member publishers when conflicts cannot be resolved. This actual imbalance of power leaves authors with little power to hold this large and powerful organization - or its members - accountable for inconsistencies in ethical policies. For example, COPE does not clearly define the ethics of self-plagiarism, although it does indicate that duplication is a form of redundancy. Rather than clearly stating what, and to what extent, self-plagiarism is accepted, or whether it is unethical or not, it leaves the power of decision in the hands of its individual member editors to figure it out, offering instead a set of simplistic flow-charts for 
editors to orientate themselves. Weak and unclear definitions by COPE and by its members - to some extent washing their hands free of this responsibility - leave authors in the dark as to what precisely is self-plagiarism, how much selfplagiarism is unethical, and if in fact it constitutes misconduct at all within the context of publishing. Although this editorial independence is a positive aspect of publishing because it allows editors to establish their own limits and parameters without a publisher's imposition, it begs the question: what then is the advantage of becoming a COPE member if only to be a member of an exclusive publishing club while having to make ethical decisions on one's own? What then is the true value of COPE membership and what services precisely are being offered to member editors and publishers of this exclusive ethics club that should not be known to the public? In other words, what exactly does COPE membership buy?

This issue and debate about the excessive ethical power conferred to paying COPE members has become even more pertinent recently. Deborah Kahn, who is the publishing director of STM journals at Taylor \& Francis / Informa, a COPE member, and also the chair of the Ethics Committee for all Taylor \& Francis journals, was recently appointed (early July, 2016) as one of the eight newly appointed members of the COPE Council. Only in the first days of September did Kahn's name officially appear on the list of COPE Council members. This is because, in July 7, 2016, a message appeared on the COPE web-site stating: "Note: Deborah Kahn (Editorial Director, Taylor \& Francis, UK) applied to be a member of COPE Council and has been elected by the General Membership. However, Deborah will not be taking up her position until an ongoing case involving Taylor \& Francis has been resolved." It was never explained to the public what this case was and if and how it was resolved, again undermining the transparency of this organization. This presents a direct ethical and financial conflict of interest and thus fortifies the notion that COPE exists almost exclusively to represent the rights and financial perspectives of its paying members using ethics as a front for the public. This bias, which is in plain sight, further erodes away at authors' rights, which are already minimal (Al-Khatib and Teixeira da Silva 2016). When authors' rights are minimized, especially when dealing with a powerful ethics organization, the opportunity to challenge editors and publishers, especially those that are COPE members, becomes reduced. Consequently, violations of codes of conduct by editors or publishers who are COPE members not only go under the radar, there are no immediate consequences for such offenders, or at least not to the public eye. The issue of the violation of COPE codes of conduct for editors has been discussed previously (Teixeira da Silva 2015a), focusing on clause 3.1, which states (COPE 2016e) that "Editors' decisions to accept or reject a paper for publication should be based on the paper's importance, originality and clarity, and the study's validity and its relevance to the remit of the journal." and on clause 15.1, which states that "Editors should respond promptly to complaints and should ensure there is a way for dissatisfied complainants to take complaints further. This mechanism should be made clear in the journal and should include information on how to refer unresolved matters to COPE."

Even though COPE represents a single organization that requests its members to have standardized policies, close scrutiny of the guidelines related to errata, expressions of concern and retractions revealed large variation among COPE member publishers (Teixeira da Silva and Dobránszki 2016). This inconsistency is extremely problematic for authors who may submit papers to different COPE members and be confronted with varying ethical definitions, potentially placing them in an ethical bind. For example, one requirement for COPE members is that retraction notices should be open access and free for the public to view. However, several cases were detected in COPE members where retraction notices lay behind a pay wall, in direct contravention of COPE policies (Teixeira da Silva 2015b). The lack of perfect consistency among paying COPE members reduces trust in their stated ethical principles and indicates that a two-tier system of ethics exists: one for paying COPE members, and another - less distinct one - for non-COPE members.

How then can authors and the scientific public hold powerful COPE member journals and editors more accountable? How can greater transparency be invoked when authors have no right of direct challenge? What are the ethics of banning scientists for making challenges to this powerful publishing status quo when no rules of engagement exist to challenge editors and publishers (Palus 2015)? And what value does COPE membership have when there is wide variation among paying COPE members in terms of specific issues of publishing ethics and a wide range of editorial independence? One of the reasons underlying the erosion of trust in science publishing lies precisely in the inconsistencies and lack of accountability by COPE, or its members, even though its purported mission is to increase standardization of ethics among its members and improve accountability to the public and authorship. The 2016-2018 COPE strategic plan (COPE 2016f) was released in the first week of September 2016, and the introductory statement by the Chair, Virginia Barbour, is extremely telling: "It is our mission over the next few years to build on this with the aim to move the culture of publishing towards one where ethical practices become the norm, part of the culture itself, not something imposed from outside." This suggests that COPE wishes to strongly implement its ethical ideas, as determined by its paying members, and that it will not consider suggestions made by non-members.

Although strong publishing ethics are required in science publishing, and a fair, unbiased and objective body that can appreciate the ethical needs and interpretations of a range of proponents, and not only COPE members, is required, 
as it currently operates - at least in the eyes of regular authors - COPE represents a strongly biased, industry-favoring ethical camouflage, without sufficient accountability to the public, either about its own operations and costs, or about the inconsistencies among its members. Science needs an ethical body - even so, should ethics be centralized in this way by such an opaque organization? - that fully understands that the authorship is the most important player in the scientific publishing landscape. Without authors, there are no papers, journals or publishers. Therefore, only interaction with authors can mould the future of academic publishing, and not the assumption of ethical superiority without equal accountability, openness and transparency.

\section{Conflicts of Interest}

The author has been banned by two COPE members, Elsevier BV's Scientia Horticulturae, and Taylor \& Francis I Informa.

\section{References}

Al-Khatib, A., Teixeira da Silva, J.A. (2016) What rights do authors have? Science and Engineering Ethics (in press). DOI: 10.1007/s11948-016-9808-8

Committee on Publication Ethics (COPE) (2016a) About COPE. http://publicationethics.org/about (last accessed: 23 November, 2016)

COPE (2016b) Promoting integrity in research publication. http://publicationethics.org/ (last accessed: 23 November, 2016)

COPE (2016c) Members. http://publicationethics.org/members (last accessed: 23 November, 2016)

COPE (2016d) Council members. http://publicationethics.org/about/council (last accessed: 23 November, 2016)

COPE (2016e) Code of conduct and best practice guidelines for journal editors. http://publicationethics.org/files /Code_of_conduct_for_journal_editors_Mar11.pdf (last revision: 2011; last accessed: 23 November, 2016)

COPE (2016f) Strategic plan 2016-2018. http://publicationethics.org/files/u7140/StrategicPlan2016_2018.pdf (last accessed: 23 November, 2016)

Larivière V, Haustein S, Mongeon P (2015) The oligopoly of academic publishers in the digital era. PLOS ONE 10(6): e0127502. doi:10.1371/journal.pone. 0127502

Palus S. (2015) Biologist banned by second publisher. http://retractionwatch.com/2015/09/24/biologist-banned-by-second-publisher/ (last accessed: 23 November, 2016)

Teixeira da Silva, J.A. (2015a) COPE code of conduct clause 3.1. under the microscope: a prelude to unfair rejections. Current Science 109(1): 16-17.

Teixeira da Silva, J.A. (2015b) Pay walled retraction notices. Bangladesh Journal of Bioethics 6(1): 27-39. DOI: 10.3329/bioethics.v6i1.24403

Teixeira da Silva, J.A., Dobránszki, J. (2016) Notices and policies for retractions, expressions of concern, errata and corrigenda: their importance, content, and context. Science and Engineering Ethics (in press). DOI: 10.1007/s11948-016-9769-y 